\title{
Médiévales
}

Langues, Textes, Histoire

45 | automne 2003

Grammaires du vulgaire

\section{Grammaires du vulgaire}

Christopher Lucken et Mireille Séguy

\section{(2) OpenEdition}

Journals

Édition électronique

URL : https://journals.openedition.org/medievales/638

DOI : 10.4000/medievales. 638

ISSN : 1777-5892

Éditeur

Presses universitaires de Vincennes

Édition imprimée

Date de publication : 16 septembre 2003

Pagination : 5-10

ISBN : 2-84292-146-1

ISSN : 0751-2708

Référence électronique

Christopher Lucken et Mireille Séguy, "Grammaires du vulgaire », Médiévales [En ligne], 45 | automne 2003, mis en ligne le 26 octobre 2005, consulté le 24 avril 2022. URL : http://journals.openedition.org/ medievales/638; DOI : https://doi.org/10.4000/medievales.638

Ce document a été généré automatiquement le 24 avril 2022

Tous droits réservés 


\title{
Grammaires du vulgaire
}

\author{
Christopher Lucken et Mireille Séguy
}

1 La "grammaire» désigne en ancien français le latin. Lui seul est pourvu d'une grammaire : celle notamment que décrivent l'Ars minor et major de Donat (IV siècle) ou les Institutions grammaticales de Priscien ( $\mathrm{vI}^{\mathrm{e}}$ siècle), à une époque où le "bon " latin (celui de la rhétorique illustrée par les meilleurs orateurs et défendue par Cicéron, et qui deviendra le latin "classique ») semble devoir s'effacer devant l'invasion du latin « vulgaire ", non seulement parlé par le " peuple » rustique et les barbares, mais aussi favorisé par les premiers chrétiens dont le sermo humilis est destiné à être entendu de tous et ne saurait plier la voix de Dieu aux règles de quelque bon usage (du moins théoriquement). L'objectif de cette institution de la grammaire comme celui, par la suite, de la réforme d'Alcuin sous Charlemagne, était, dans la mesure du possible, de fixer la langue, de freiner les transformations qu'elle pouvait subir d'une région à l'autre de l'empire latin ou à travers le temps, à l'instar de ce que l'écriture - la litteratura au sens littéral du terme - est capable de réaliser. Il s'agit en quelque sorte de soumettre le langage à la rectitude de la lettre, de la littera (ou de la gramma), comme aux directives des litterati, des clercs, et de conserver ainsi la mémoire inaliénable de son identité.

2 Si le latin officiel de l'administration et du pouvoir étatiques ou ecclésiastiques est défini par la norme grammaticale, le latin vernaculaire parlé par le «vulgaire » est perméable à la variation. C'est de ce dernier que va naître cette lingua romana rustica que le Concile de Tours (en 813) préconise aux prêtres d'employer lors de la prédication, à la place du latin, afin d'être compris des fidèles : soit, ce qui va devenir, pour la future France, le roman puis le françois. Cette nouvelle langue, cependant, ne cessera d'évoluer tout au long du Moyen Âge. Divisée en plusieurs dialectes, elle paraît en outre sensiblement différente selon les régions où elle est parlée. C'est au point qu'il est souvent difficile de lui attribuer une véritable unité formelle à travers le temps et l'espace. Mais est-elle pour autant étrangère à toute grammaire?

Le premier texte en langue «française ", les Serments de Strasbourg (842), contenu dans l'Histoire des divisions entre les fils de Louis le Pieux de Nithard, témoigne à la fois de l'institution du français comme critère identitaire fondamental d'une nation française encore en gestation (à l'occasion de la division du territoire de Charlemagne entre la 
partie francophone attribuée à Charles le Chauve et la partie germanophone attribuée à Louis le Germanique) et de l'institution de la langue française dans l'espace habituellement régi par le latin littéraire et ses règles propres : l'écriture ${ }^{1}$. Ce qui ne sera pas sans conséquences. Si la langue latine s'ouvre ainsi à la multiplicité potentielle des langues vernaculaires (représentées ici par la romana lingua et la teudisca lingua), soit à une forme de babélisation, et finira par perdre son statut de langue commune à l'ensemble de la chrétienté, un tel geste n'est pas sans effet en retour sur la nature même de cette nouvelle langue. Celle-ci n'est plus seulement une langue vernaculaire : elle devient désormais une langue « littéraire ». Il lui faut s'inventer une graphie et, du même coup, une sorte de grammaire. Comme le montrent en effet les différentes études recueillies ici, le passage à l'écriture de la langue vulgaire la confronte inéluctablement aux principes de régularité exigés par la grammaticalité de la litteratura. Si ceux-ci peuvent se modifier, ils n'en sont pas moins à l'œuvre dans le travail des scribes. Pourtant, ils ne seront pas formalisés par des règles strictes et constantes avant la fin du Moyen Âge. Il faudra attendre la Renaissance pour qu'on légifère sur la langue française afin d'en codifier l'emploi de façon contraignante. Qu'en est-il alors, plus précisément, de cette grammaire, ou plutôt de ces grammaires - plurielles - du vulgaire?

4 Dans son Éloge de la variante, B. Cerquiglini constate que « pour se fonder comme science au XIX ${ }^{e}$ siècle, la linguistique dut se donner, par réduction, un objet stable et simple, régulier, homogène. De ce point de vue, l'ancien français, tel qu'il apparaît aux yeux des savants positifs qui entreprennent de le décrire, semble marqué d'une hétérogénéité constitutive. Car, à la variance textuelle incessante qu'offrent les manuscrits, s'ajoute l'infinie variation des formes du langage $»^{2}$. Plutôt que de chercher à dégager les raisons de ces variations et d'en découvrir la cohérence éventuelle, la philologie romane s'efforce de l'éliminer au profit d'une description, fondée sur la grammaire et la phonétique historiques principalement, susceptible de retracer une évolution régulière et homogène de la langue située entre la grammaire latine et celle du français moderne ${ }^{3}$. Il s'agit d'en restituer ainsi l'unité afin de la pourvoir à son tour d'une grammaire.

Un tel objectif trouve son monument exemplaire dans La Vie de saint Alexis publiée par G. Paris en 1872. Non seulement l'édition de ce texte du xi ${ }^{\mathrm{e}}$ siècle "a pour but de retrouver, autant que possible, la forme que l'ouvrage [...] avait en sortant des mains de l'auteur", mais aussi, en en reconstruisant l'original, de lui restituer une langue uniforme et régulière, enfin débarrassée des irrégularités et des "fautes » que les scribes anglo-normands avaient pu y ajouter, et de proposer du même coup «un spécimen admissible de la bonne langue française telle qu'elle devait se parler et s'écrire au milieu du XI $\mathrm{e}^{\mathrm{e}}$ siècle $»^{4}$. C'est à l'aune de ce modèle textuel, longtemps destiné à servir de texte de référence pour l'enseignement de la grammaire de l'ancien français, que G. Paris peut découvrir avec surprise, dans La Vie de saint Gilles de Guillaume de Berneville (xII ${ }^{\mathrm{e}}$ siècle), "une langue aussi correcte, et notamment un vocalisme aussi pur et aussi nuancé chez un auteur anglo-normand de la fin du règne de Henri II ", dont le langage, comparé à d'autres, s'avère « bien supérieur, c'est-à-dire bien plus fidèle aux lois traditionnelles du français de France $»^{5}$. C'est ce qui permet aussi, par exemple, à P. Meyer de qualifier la langue teintée de dialecte employée en Angleterre par Nicole Bozon dans ses Contes moralisés (xIV siècle) d'« idiome très corrompu » : « La langue qui s'était conservée dans un état de pureté relative jusqu'aux 
premières années d'Henri III, dégénère rapidement avant le milieu du XIII ${ }^{e}$ siècle ». Au lieu d'être " uniforme ", " elle offre au contraire dans sa corruption une variété assez grande $»^{6}$. Au regard de la norme, construite pourtant de façon artificielle, la variation est considérée comme une défaillance qu'il n'est pas nécessaire de prendre en compte pour réfléchir à l'histoire de la langue et à la constitution de son système.

Comme on peut le constater avec ces derniers exemples, l'histoire de l'ancien et du moyen français ne s'arrête pas avec la fin du Moyen Âge. Elle ne cesse de se poursuivre à travers ceux qui cherchent à décrire cette langue et qui la constituent dès lors dans son identité en même temps qu'ils en font un objet d'étude. Depuis une trentaine d'années, l'ancien français a bénéficié des apports de la linguistique structurale et de la sociolinguistique qui ont permis de mieux comprendre la diversité de ses formes et les " processus de grammatisation » qui en caractérisent l'histoire. Plutôt que de blâmer la langue dégradée ou fautive de quelque scribe ignare, inattentif ou marqué par son parler local, ces nouvelles approches ont su donner sens et, même, attribuer une certaine cohérence logique aux écarts qui viennent contredire la série de règles que l'on avait pu déduire des meilleurs textes ${ }^{7}$. Pourtant, à bien des égards, l'enseignement de la langue française du Moyen Âge dans l'université française est restée tributaire des présupposés hérités de la tradition philologique $\mathrm{du}$ xIX ${ }^{\mathrm{e}}$ siècle, pour laquelle l'ancien français, alors même qu'il était traité dans une perspective diachronique (conformément à la grammaire historique alors dominante), se pensait essentiellement dans la perspective de la constitution d'une langue nationale aux normes fixes, immuables et, plus ou moins secrètement, universelles (soit cette "bonne langue française ", dont parle G. Paris). Cela tient non seulement à une certaine conception traditionnelle de la langue française, celle du bon usage qui la veut claire, pure et rationnelle, mais aussi, nous semble-t-il, à la nature même d'un enseignement étroitement assujetti à la préparation des concours d'enseignement, CAPES et agrégation, et à leurs exigences singulières qui privilégient un savoir normatif aisément mémorisable. La grammaire sert de fondement à l'école, et inversement. Cet état de fait est doublement dommageable: dommageable pour la langue médiévale, mais aussi pour la littérature médiévale.

7 Pour la littérature: les textes mis au programme sont pour la plupart des textes littéraires majeurs. Or ces textes ne sont abordés par la majorité des étudiants (ceux qui préparent le CAPES ou qu'on prépare dès la première année à préparer le CAPES) que comme les supports d'exercices techniques (phonétique historique, morphologie, syntaxe, fiches de vocabulaire), situation aussi absurde que celle qui consisterait, pour un étudiant ne connaissant rien ou pas grand-chose au roman du xix siècle, à aborder par exemple Madame Bovary uniquement par le biais d'exercices de grammaire. Le risque réel de cette entreprise de réduction d'un corpus littéraire à un terrain d'exercices d'école est de faire disparaître tout intérêt éventuel pour la littérature médiévale, et même de faire perdre de vue qu'une telle littérature existe, que l'on peut interroger le fait littéraire médiéval au même titre que l'on interroge le fait littéraire contemporain - même s'il peut relever de problématiques autres et soulever des enjeux différents.

Mais la manière dont on aborde aujourd'hui le Moyen Âge dans les études de lettres nuit aussi à la langue médiévale elle-même. Le français médiéval, tel qu'il est enseigné habituellement à l'université, souffre d'un manque de réflexion sur la place qu'on désire lui assigner, tant dans la perspective de la lecture des textes médiévaux, dont il 
est trop souvent déconnecté, que dans la perspective d'une histoire de la langue française. Sur ce dernier point, on ne peut que constater l'écart que l'enseignement institutionnel de l'ancien français creuse aujourd'hui entre une langue pour une part reconstruite, dont on tente de montrer qu'elle obéit à des règles strictes préparant en quelque sorte l'émergence d'un français contemporain lui-même considéré comme monolithique et homogène, et d'autre part la réalité mobile et polymorphe de la langue médiévale. Or, resituer la langue médiévale dans une évolution longue, où prédominent les tensions entre variations et normalisations, mettre en avant l'importance des relations qu'elle a pu entretenir avec les langues qui lui ont préexisté et avec lesquelles elle a coexisté, tenter d'apprécier l'enjeu qu'a pu représenter l'institution du français dans la formation des identités nationales, c'est aussi, sans aucun doute, se donner les moyens de comprendre mieux ce qui se joue dans les évolutions et les variations du français, de sa naissance à la période contemporaine ${ }^{8}$. L'enseignement institutionnel du français médiéval s'avère incapable d'aborder de telles questions lorsqu'il se contente de transmettre des recettes et des modes d'emploi pouvant être facilement retenus par les étudiants afin qu'ils réussissent aux concours d'entrée à l'enseignement dans les conditions qui seront les leurs le moment venu. En privilégiant une approche sociolinguistique, en soulignant la manière dont la langue génère sa propre transformation au moment même où elle participe des transformations sociales, politiques et culturelles, les études recueillies ici interrogent la façon dont la langue française a fait l'histoire et l'histoire la langue française : soit comment le français s'est fait dans l'histoire.

9 Il ne s'agit pas pour autant de récuser toute approche synchronique de la langue médiévale et toute grammaire de l'ancien français9. Au contraire, comme on pourra s'en rendre compte en lisant les études qui suivent, les processus de grammaticalisation sont à l'œuvre dans la lingua romana rustica, dans cette langue vulgaire qui allait devenir le «français de France ", dès sa première apparition (avec les Serments de Strasbourg), et se manifestent dans tous les domaines et à tous les moments de son histoire. Les différentes analyses grammaticales de l'ancien français sont donc parfaitement légitimées à vouloir dégager les règles capables d'expliquer son fonctionnement et son usage. Mais là où la grammaire historique traditionnelle subordonne sa description à la norme dont elle s'évertue à mettre en évidence l'apparition (et la dégradation inéluctable), l'approche structurale construit une combinatoire dont les mécanismes pouvaient être perçus par les scribes ou locuteurs contemporains, au sein d'un système à même de générer - ou de gérer - sa propre temporalité.

Si l'enseignement de la langue du Moyen Âge a du mal à trouver sa place, c'est aussi, plus profondément peut-être, parce que cette langue, tout comme la littérature médiévale, ne vont pas de soi. P.Zumthor, parmi les premiers, a souligné la place singulière qu'occupe la période médiévale dans l'imaginaire collectif contemporain ${ }^{10}$. Si d'un côté le Moyen Âge appartient bien à notre histoire, dont il signe d'un certain point de vue l'émergence, si la langue et la littérature françaises vernaculaires naissent effectivement à cette période, il peut sembler d'un autre côté beaucoup plus éloigné de notre fonds commun culturel que ne l'est l'Antiquité gréco-latine. On connait (on connaissait) généralement mieux la littérature et l'histoire antiques que la littérature et l'histoire médiévales. Ou, du moins, et c'est là l'important, on s'y reconnaît mieux, et nous restons en cela les héritiers directs d'une pensée de la modernité qui, depuis la «Renaissance » du XVI ${ }^{\mathrm{e}}$ siècle, s'est pour beaucoup définie en termes d'opposition à la période médiévale, dès lors constituée comme époque des Ténèbres, ou à tout le moins 
comme époque de l'altérité, de ce que l'on n'est pas. Le lieu commun « on n'est pas au Moyen Âge » ne doit-il pas aussi s'entendre comme un " on ne naît pas au Moyen Âge ", l'identité française préférant s'enraciner ailleurs, c'est-à-dire en l'occurrence dans l'Antiquité ? Il en est de même pour la langue : à la variation du français médiéval, on préfère la norme grammaticale et atemporelle du latin classique, fût-elle en partie fantasmatique pour avoir été réduite à la langue immuable de "Cicéron ». Ce rapport contradictoire et inconfortable que nous entretenons avec le Moyen Âge, cette difficulté que nous éprouvons à établir la juste distance avec lui ressortissent sans doute aux liens problématiques que l'on peut entretenir avec ses origines ou avec ce qui en tient lieu pour nous. C'est sans doute là que réside essentiellement aujourd'hui l'étrangeté médiévale, telle qu'elle se devine notamment dans les rapports problématiques que nous entretenons avec la langue de cette période, notre langue maternelle en voie d'émergence et d'identification ${ }^{11}$.

\section{NOTES}

1. Cf. R. Balibar, L'Institution du français. Essai sur le colinguisme des Carolingiens à la République, Paris, 1985, et B. Cerquiglini, La Naissance du français, Paris, 1991.

2. B. Cerquiglini, Éloge de la variante. Histoire critique de la philologie, Paris, 1989, p. 87.

3. $C f . S$. Branca, «Les débats sur la variation au milieu du XIX siècle ", Recherches sur le français parlé, 5, 1983, p. 263-90.

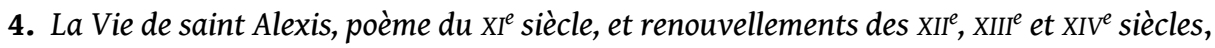

G. Paris et L. Pannier éd., Paris, 1872, p. 8 et 135.

5. La Vie de saint Gilles par Guillaume de Berneville, G. Paris et A. Bos éd., Paris, 1881, p. xxxv.

6. Les Contes moralisés de Nicole Bozon, frère mineur, L. Toulmin Smith et P. Meyer éd., Paris, 1889, p. lvii-lviii.

7. Cf. S. Auroux, «Le processus de grammatisation et ses enjeux », en introduction à Histoire des idées linguistiques, S. Auroux dir., t. II, Le Développement de la grammaire occidentale, Paris, 1992, p. 11-64. Pour une réflexion méthodologique sur l'ancien français, $c f$., notamment, J. Batany, « Ancien français, méthodes nouvelles ", dans Histoire de la langue, A. Lerond éd., Paris, 1971, p. 45-50 ; R.-L. Wagner, L'Ancien Français. Points de vue, programmes, Paris, 1974 ; B. Cerquiglini, J. Cerquiglini, C. Marchello-Nizia et M. Perret, «L'objet "ancien français" et les conditions propres de sa description linguistique ", dans Méthodes en grammaire française, J.-C. Chevalier et M. Gross éd., Paris, 1976, p. 185-200 ; C. Marchello-Nizia, "Questions de méthode », Romania, 106, 1985, p. 48392 ; S. Fleischman, « Medieval Vernaculars and the Myth of Monoglossia. A Conspiracy of Linguistics and Philology ", dans Literary History and the Challenge of Philology. The Legacy of Erich Auerbach, S. LERER éd., Stanford, 1995 ; ID., « Methodologies and the Ideologies in Historical Grammar ", dans Medievalism and the Modernist Temper, R. H. Bloch et S. G. Nichols éd., Baltimore-Londres,1996, p. 402-38.

8. Parmi les histoires récentes de la langue française qui rejoignent d'une manière ou d'une autre notre propos, $c f$. J. Picoche et C. Marchello-Nizia, Histoire de la langue 
française, Paris, 1989 ; R. A. Lodge, Le Français. Histoire d'un dialecte devenu langue [1993], Paris, 1997 ; M. Perret, Introduction à l'histoire de la langue française, Paris, 1998 ; C. Marchello-Nizia, Le Français en diachronie : douze siècles d'évolution, Paris, 1999 ; et Nouvelle histoire de la langue française, J. Chaurand dir., Paris, 1999.

9. Cf., notamment, C. Buridant, Grammaire nouvelle de l'ancien français, Paris, 2000. 10. Cf. P. Zumthor, Parler du Moyen Âge, Paris, 1980.

11. Les études recueillies dans ce numéro ont été présentées dans le cadre des Ateliers de Médiévales le 8 novembre 2002 au Musée d'Art et d'Histoire de Saint-Denis. Cette rencontre a pu se réaliser grâce au soutien financier des équipes de recherche Littérature et histoires (Upres/ead, Université Paris 8), Études et éditions de textes médiévaux (Upresa 8092, Université Paris IV), et Diachronie du français et évolution des langues (GDR 2349, Université-CNRS). Que leurs directeurs respectifs, Monsieur Jacques Neefs, Mesdames Jacqueline Cerquiglini-Toulet et Christiane Marchello-Nizia, en soient vivement remerciés, ainsi que la directrice du Musée d'Art et d'Histoire de Saint-Denis, Madame Sylvie Gonzalez. L'intervention de Michèle Perret, « Approche synchronique vs Approche diachronique. Un exemple, la déclinaison ", n'a malheureusement pas pu être recueillie ici.

\section{AUTEURS}

\section{CHRISTOPHER LUCKEN}

Département de Littérature française, Université Paris 8-Vincennes/Saint-Denis, 2, rue de la Liberté, F-93526 Saint-Denis Cedex

\section{MIREILLE SÉGUY}

Département de Littérature française, Université Paris 8-Vincennes/Saint-Denis, 2, rue de la Liberté, F-93526 Saint-Denis Cedex 